\title{
A Conversation with Elisabeth Niklasson
}

Elisabeth Niklasson is lecturer at the School of Geosciences at the University of Aberdeen. She holds two master's degrees in Cultural Heritage and Computational Archaeology from Gothenburg University, and a PhD in Archaeology from Stockholm University. She has long been interested in the ethical and political aspects of archaeological heritage, particularly relating to contemporary identity politics. A core part of her research has focused on European heritage policies, studying the influence of European Union (EU) funding schemes on archaeology. She has also reflected critically on the reception of aDN $A$ in archaeology. In recent years, she has expanded her focus to analyse uses of the past by populist and extreme right movements in Europe, making vital contributions to our understanding of heritage in times of political polarization.

Martina Revello Lami: Let's start from the beginning. Can you please tell us how your interest in European Union heritage policy and grants started? How did you become interested in the policies behind the mechanisms of funding in Archaeology?

Elisabeth Niklasson: Thank you Martina and thank you for this question. To make a long story short, it started during my masters, when I came upon some online funding applications of the EU culture programs. These programs have specific research questions, and among them, there was one aiming to prove the existence of a European identity in the past by way of heritage and archaeology. It seemed as if they had already found the answer they were looking for, like they had invented this idea of a European people in the past to fit the current EU funding framework, as a sort of politics applied to archaeology.

I became interested in understanding how that happened, so I started to look at the mechanisms for how funding drives specific interests in archaeology, and how it influences some of the research questions we ask.

We often get encouraged to write in a certain way, to write "application poetry" as some archaeologists I have interviewed calls it, and I wanted to know how this application poetry worked in the EU funding circles. What actually came out of those projects where they wrote that they would try to find a European identity in the past? So I researched this for several years as part of my $\mathrm{PhD}$.

In our conversations ahead of the interview, you mentioned my critical stance in regards to the ancient DNA (aDNA). I did not look specifically into such questions during my $\mathrm{PhD}$ research, or all of these modern trends that we see right now on ancient migrations, tightly connected to the European grant narratives. But I remember finding this big project called "Forging Identities" about migration and the population of Europe in the Bronze Age. I saw how easy it seemed to get money when you started to ask questions regarding origins, migration, and particularly aDNA. 
Martina Revello Lami: The aDNA revolution has redefined human origins, health and mobility, and established a new prehistory. In your work you have prompted archaeologists to embed more firmly critical thinking into genetic data and challenge deepseated assumptions linking biology to culture. A plea for complexity not always taken up by scholars and professionals, yet genetic research is most popular right now and much funding is directed in that way. Why?

Elisabeth Niklasson: Archaeologists have always been excited about new things.

When radiocarbon dating came along, everyone was happy to start to integrate it in different ways (although there was a debate behind that as well). As soon as we get new tools to use with the extremely fragmented materials that we have to deal with every day, we want to implement them because we really want to know what happened in the past. We do our very best to find the best explanation we can, and I think there is a genuine curiosity and interest in how we can implement new tools and new resources.

aDNA was introduced at a critical political juncture when western societies started to turn inwards again, towards questions of origins. In many societies, there was a sort of a backlash against this idea about plurality and multiculturalism, and a renewed interested in the origins of European peoples. aDNA became popular at the same time this happened.

The question of where we come from has always been a dangerous one, in many ways, but also something we, as archaeologists, are very interested in. I think it is fine to ask it, but we need to be aware that it is not an innocent question. Ancient origins still matter. If you are trying to enter Europe as a refugee along the Mediterranean coast, for instance, being asked about your heritage or where you come from could be a matter of life and death.

When biologists started to work with archaeological materials, and when archaeologists started to use aDNA sequencing results to answer specific questions about the origins and migrations of people, this became a real moneymaker and attention grabber. Within academic politics and funding schemes, it became an area where this 'application poetry' could be readily applied. Now, with the latest techniques, such as next-generation sequencing and the use of the small petrous bone to provide DNA, researchers can get better results than before, and merging questions of origins and aDNA have become a potent cocktail that attracts funders, because it is easy to grasp even if you have no knowledge about archaeology.

Such studies also have great success in high-impact journals, because aDNA is seen as a truer science than the critical humanities sciences - as a science where you can get clear results. Some proponents in our field have even suggested, like Kristian Kristiansen, that this means we don't have to rely on "guesswork" anymore. That we can know for real where past peoples came from and how migrations worked. Finally, there is the narrative of the third science revolution in archaeology. All of these ingredients have come together in this potent cocktail, making aDNA the new shiny thing for funders to fund and for archaeologists to focus on.

Either you are on the aDNA train, it seems, or you are left in prehistory, literally. There is less chance of gaining funding or being part of new developments in archaeology if you stay on the critical humanities side; that is how it has been portrayed. 
Martina Revello Lami: The so-called "third science revolution" has impacted archaeology in all its sectors. Is there a difference in how genetic data has changed the work of archaeologists in research, museums, rescue archaeology and more widely the commercial sector? For instance, the relationship between migration and material culture has been and still is the subject of a heated debate. How can we communicate the complexity of aDNA data to the public? Can we disseminate this critical thinking to a larger audience?

Elisabeth Niklasson: I would say that on a day-to-day sort of basis, the "third science revolution" has not really changed what we do that much. It has certainly brought many new possibilities and new ethical considerations to the table, such as the destructive sampling for aDNA research and the impact on contemporary communities. Museums and collections have been unsure if it is worth destroying bones for sampling purposes, and of how they should deal with all the requests that come in from researchers? This has directly affected the work of curators.

It has also led to questions about the risks involved when working with material linked to indigenous or minority populations. How do archaeologists assess the impact of their results on living people today? In many cases, such as with Native American peoples, claims to land and other legal rights are dependent on the ability to prove continuity and genetic ancestry. What if the DNA results are different from what was expected? How will that affect the contemporary population?

If you are a field archaeologist, you now have to consider whatever you excavate will be tested for aDNA, and think about how this might affect the people you are working with. How can we communicate aDNA results to the public? I think it is tricky, and it will be tricky for archaeologists for a long time, because it is a really alluring topic and easy to fall into the trap of telling people about what they might want to hear: did they look like us? Did they have the same skin colour or the same eye colour as us? Even though aDNA can sometimes answer these questions, we have to ask ourselves: why do we want to know what kind of eye colour they had? How will this say anything about the ancient peoples we study? What can it tell us about their societies?

When it comes to our interactions with mass media, especially when they seek to publish on topics like aDNA, I think we should approach it almost as politicians do in election campaigns: speak to the undecided. We are not here to convince those who already have a clear idea of the past. People who watch documentaries all the time and who know about archaeology, they already have a pretty clear idea of what the past is about. Similarly, people with a strong identity as 'Viking' descendants, for instance, are already set in their beliefs. Then there are people who are not interested in prehistory or heritage, and that is ok. Not everyone has to care about heritage and archaeology. It is a sort of "tyranny of participation" to try to make everyone love archaeology and be part of it. We do not have to preach to people who are uninterested. Instead we should speak to the undecided, to those interested publics who follow archaeological news and tell their kids about new discoveries. If we can raise more critical aspects and work more sustainably with how we distribute our information in media directed to those groups, we can really make a change. 
Martina Revello Lami: A growing number of European grants are financing research based on aDNA analysis to trace human mobility. What do you think will be the longterm effect of such funding policies on our discipline? Will it be beneficial for archaeologists at all latitudes, or will it deepen differences by creating an even greater divide between archaeologists working in the field and those carrying data analysis and crafting overarching narratives? Are we still on time to reverse such trend?

Elisabeth Niklasson: I think there is some potential to reverse the trend. I think there are incentives now, and there are ongoing discussions that are starting to become more and more nuanced.

In the last years, many people have been taking sides: there are people who are pro aDNA research and people who are skeptical. However, a couple of things need to happen.

First, we need to embed a sense of ethics in all aspects of working with aDNA. To have an idea of what you want to know before asking for bone samples, not just collecting and testing it all. We also need to integrate aDNA research within archaeological thinking and practice. It is like a puzzle piece that does not quite fit yet. I think we need to figure out where it fits in the history of the archaeological discipline. Is it just another method? We make comparisons with radiocarbon dating, but there is no field working only with that. We have to figure out how to integrate it, and this takes a long time. We cannot really expect it to change in the coming years, but I think these more nuanced discussions will help. There will hopefully be more interest among population geneticists to understand the theoretical basis of archaeological reasoning. We have to work with both theory and methods and figure out how they fit together. It is a general answer, but I think there are many ways to do this.

In my research, when I talk about funding and politics, I always say that we need to work better across sectors. Academia really needs to be better engaged with the professional heritage sector, and all the way down to excavations and contract archaeology as well.

Martina Revello Lami: You had direct experience with the administration of European grants. Can you tell us how the administration of these grants actually work?

Elisabeth Niklasson: My research results were interesting. My conclusion was that even if it is just "application poetry", what you write in a grant application matters. If we write something just to get funding, and we basically copy-paste our application poetry into the final report to the funder, which I realized often happens, that has effects. Seeing from the inside how policy develops in the field of culture in the EU made me realize that the administration rely on those reports to see if their money renders the kind of research they were looking for, and that we need more of need more of. And based on some reports, archaeology could supposedly prove a European identity in the past.

I looked at 160 successful grant applications by heritage professionals and archaeologists in the EU culture program, and I took part in the funding evaluation panels in Brussels as an observer. There, experts decided which grants got funding and which did not. I realized that it was sometimes a bit haphazard. It was not uncommon that an application got a reviewer without any expertise in the field concerned. So sometimes, you get someone from the theater or from the culture sector evaluating archaeology, and sometimes you 
get someone from geography. And if they read something that talks about aDNA, migrations, and origins for instance, they might think: «That is great, let's do that! » If you have someone from postcolonial studies or anthropology, and they get an aDNA proposal to review, they might be very critical, and they might not give it a very high score. In addition, the time pressure was very high for the reviewing process, and all the experts did this work on top of their normal work. It is not like an elitist or fancy thing to do, being a reviewer in Brussels is quite stressful: you have a certain number of proposals to review, hundreds of pages to go through. You do not have time to look into anything more than if the project has a really good idea or not.

Martina Revello Lami: Definitely an eye opener! Thank you again Elisabeth for joining us today! 